\title{
Aerodynamic jump: A short range view for long rod projectiles
}

\author{
Mark Bundy \\ US Army Research Laboratory, AMSRL-WM-BC, \\ Aberdeen Proving Ground, MD 21005-5066, USA
}

It is shown that aerodynamic jump for a nonspinning kinetic energy penetrator is not - as conventional definitions may infer - a discontinuous change in the direction of motion at the origin of free flight, nor is it the converse, a cumulative redirection over a domain of infinite extent. Rather, with the aid of an alternative kinematical definition, it is shown that aerodynamic jump for such a projectile is a localized redirection of the center-of-gravity motion, caused by the force of lift due to yaw over the relatively short region from entry into free flight until the yaw reaches its first maximum. A rigorous proof of this statement is provided, but the primary objective of this paper is to provide answers to the questions: what is aerodynamic jump, what does it mean, and what aspects of the flight trajectory does it refer to, or account for.

\section{Introduction}

The motion of a projectile can be separated into two general regions: the free-flight, FF, region and the launch-disturbance, LD, region (prior to FF). For instance, if the projectile is a saboted long rod (or, kinetic energy, KE) penetrator, then the LD region begins inbore and extends downrange to the point where shock waves from the discarding sabot petals no longer interact with the rod. The end of the LD region marks the beginning of the FF region, where the phenomenon known as aerodynamic jump (AJ) occurs. The KE penetrator is chosen to facilitate the ensuing discussion and illustrations on the subject of AJ.

Although AJ occurs in the FF region, its magnitude is influenced by events that take place in the LD region. Hence, a brief discussion of LD effects is in order. A KE projectile consists of a long rod with an aerodynamically shaped nose and stabilizing tail fins. The high mass-density, sub-caliber rod is held centered in the gun bore by a low mass-density, full-caliber sabot. The rod can undergo small, lateral, center-of-gravity
( $c g$ ) displacements and rotations while being propelled longitudinally down the bore. Such in-bore motion permits the projectile $c g$ to exit the barrel with a velocity vector oriented at an angle $\angle C G$ with respect to the instantaneous bore axis. In addition to the rod moving relative to the bore axis, the barrel itself can be moving. Thus, the rod can be launched with the instantaneous pointing angle of the bore axis, $\angle P A$, different from the original muzzle sight line. Furthermore, the instantaneous bore axis can have a lateral (crossing) velocity that is transferred to the projectile $c g$ motion. The angular change in the projectile $c g$ velocity due to this barrel crossing motion is denoted by $\angle C V$. Outside the gun, it is possible for asymmetric sabot discard to create uneven mechanical and aerodynamic forces on the rod that add yet another transverse $c g$ velocity component, and redirection angle, $\angle S D$. The net effect of these four pre-free-flight LDs can give the projectile $c g$ a cumulative transverse deflection angle, $\angle L D=\angle C G+\angle P A+\angle C V+\angle S D$, at the point where it enters FF, Fig. 1. (Bornstein et al. [1] discuss techniques to measure these LD components.)

After traveling through the LD region, the KE rod enters FF. The motion of the projectile in FF is influenced by time-dependent side forces that cause the projectile $c g$ to oscillate (swerve) about a mean FF path (swerve axis) as it travels to the target, Fig. 2. For a typical $\mathrm{KE}$ rod (which is statically stable, near-symmetric, and virtually nonrolling), the swerve curve can be approximated by a damped sine wave in both the vertical and horizontal directions.

As the trajectory of Fig. 2 is extended to a distant target, the swerve amplitude decays to near zero and the point of impact lies close to the axis of swerve symmetry, Fig. 3. (Note, the effects of gravity and the Coriolis force on the trajectory are not included in this discussion because they are not aerodynamic in nature; if warranted, their influence can simply be superimposed on the swerve motion.)

Thus, the axis of swerve symmetry has special significance because it is closely aligned with the point of impact on a distant target. As indicated by Figs 2 and 


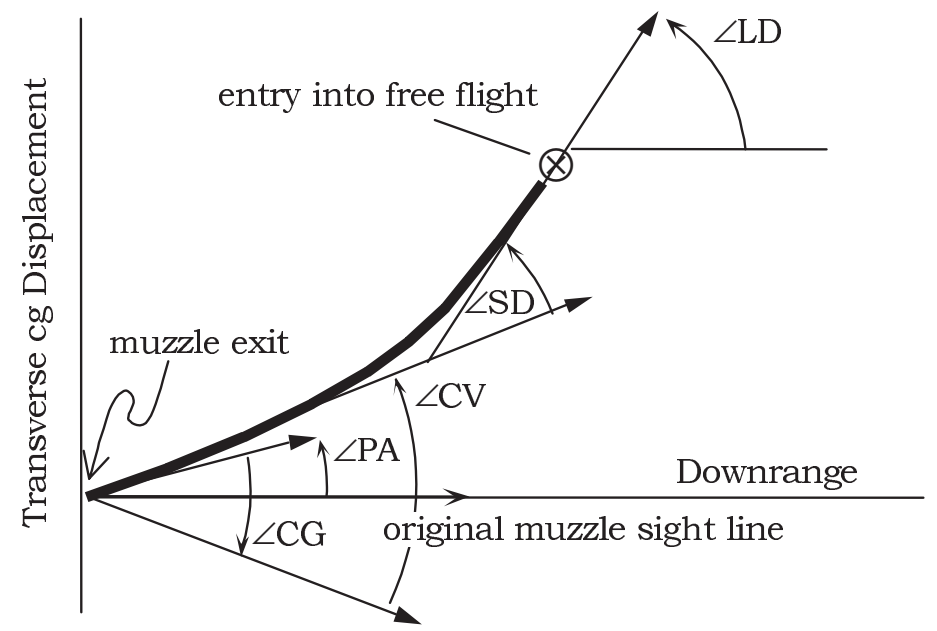

Fig. 1. Hypothetical planar CG motion of a KE rod caused by launch disturbances.

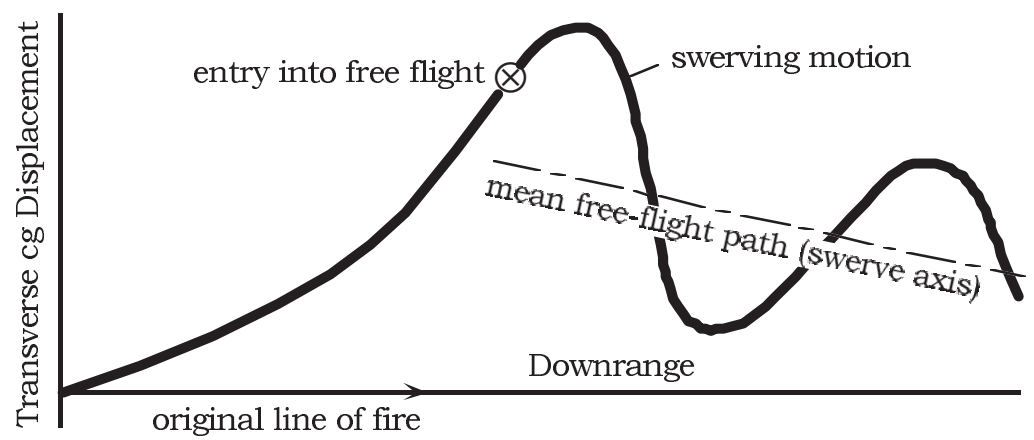

Fig. 2. Transition of CG trajectory into free flight.

3, the swerve axis can be, and most often is, different from the direction given to the projectile $c g$ as it leaves the LD region. This change in direction is caused by FF aerodynamic side forces. The term "aerodynamic jump", AJ, is used to quantify this change in direction.

One of the earliest descriptions of AJ was given by Murphy [2], stating that AJ is "the angle between the bore sightline and the 'average' trajectory when other contributors to jump are neglected". Although this definition describes AJ as an angle, it is actually the tangent of the described angle. However, for small angles (typical for AJ), the angle and its tangent are nearly one and the same. Neglecting other contributors to jump, as per Murphy [2], would mean setting, or assuming, $\angle L D=0$ in the previous discussion. In this case, Fig. 3 would transform into Fig. 4.

From Fig. 4, when the FF trajectory approaches infinity, $\mathrm{AJ}$ and $\angle A J$ can be defined by

$$
A J=\lim _{z \rightarrow \infty}\left[\frac{y}{z}\right]
$$

$$
\angle A J=\tan ^{-1}\{A J\} \underset{A J, \angle A J \ll 1}{\approx} A J,
$$

where $y$ represents the transverse $c g$ displacement and $z$ represents the longitudinal, or downrange, displacement. (Note, for later reference, the sign convention for the direction of positive $y$ in Eq. (1) will determine the sign convention for positive AJ.) Both Murphy [2] and Murphy and Bradley [3] begin their discussions of AJ based on Eq. (1). A more inclusive expression for AJ, one that does not neglect "other contributors to jump", is put forth later by Murphy [4]. This more general definition states:

$$
\begin{aligned}
& A J=\lim _{z \rightarrow \infty}\left[\frac{y-y_{o}}{z-z_{o}}\right]-\left.\frac{d y}{d z}\right|_{z_{o}} ; \\
& \angle A J \underset{\lim _{z \rightarrow \infty}\left[\frac{y-y_{o}}{z-z_{o}}\right],\left.\frac{d y}{d z}\right|_{z_{o}} \ll 1}{A J,}
\end{aligned}
$$

where $y_{o}$ is the transverse $c g$ displacement and $d y /\left.d z\right|_{z_{o}}$ is the tangent to the $c g$ displacement, both at the origin of FF. Figure 5 (an annotated version of Fig. 3) gives the geometrical interpretation of Eq. (2). 


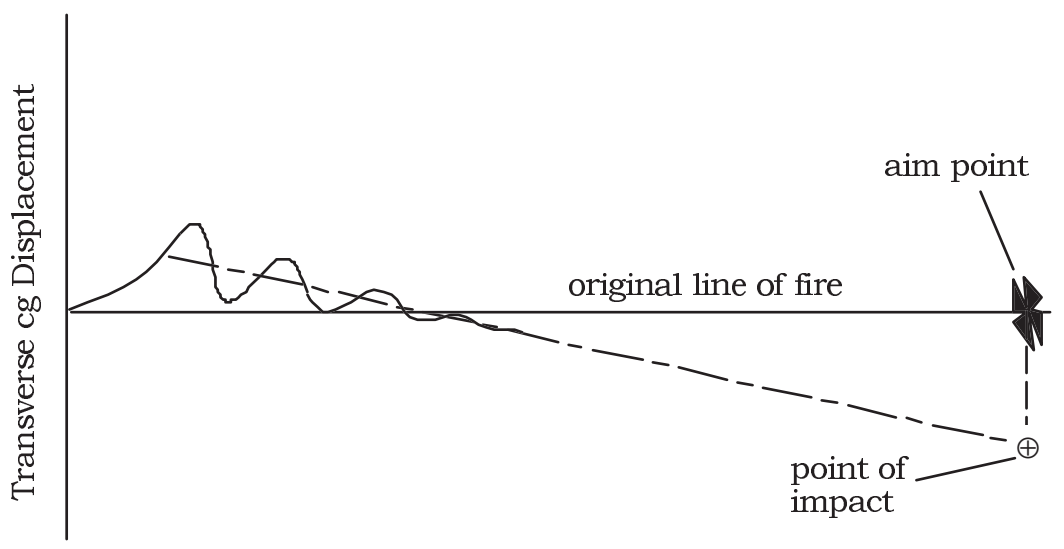

Fig. 3. Hypothetical CG trajectory extended to the target.

Equations 1 and 2 are kinematical definitions for AJ. Their more familiar dynamical counterparts are derived from them by solving, then substituting for $y$ and $z$ based on the equations of motion (as done later in this paper). Although the dynamical expressions for AJ may be more useful in practical applications, the simple geometry-based definitions of Eqs (1) and (2) are the most helpful for those seeking to visualize the effect of AJ on the $c g$ trajectory. However, because the definitions in Eqs (1) and (2) call upon the limit as the trajectory approaches infinity, some may erroneously infer from this that AJ is an effect that "accumulates" with downrange distance. In other words, they may wrongly assume from these infinity-based definitions that it takes a near-infinite amount of space to establish the AJ. The purpose of this paper is to dispel any such notions by showing that the bending of the "average" trajectory, referred to as AJ, is due to aerodynamic forces acting over a relatively short segment of the FF trajectory. This will be done, in triplicate, by first giving an alternative geometry-based, or kinematical definition for $\mathrm{AJ}$ (one that does not invoke a limiting case). Then, a physically sound explanation, supporting this definition, is given. And finally, the theoretical consequences promulgated from the alternative kinematic definition are shown to produce the same dynamical expression for AJ as that derived from Eqs (1) and (2).

\section{An alternative kinematical definition for $\mathbf{A J}$}

From Eq. (2) and Fig. 5, $\angle A J$ is the angular change between the tangent to the $\mathrm{cg}$ trajectory at the transition point between the $\mathrm{LD}$ and $\mathrm{FF}$ regions (oriented at $\angle L D$ with respect to the original line of fire) and the axis of swerve symmetry. As shown in Fig. 6, and proven in

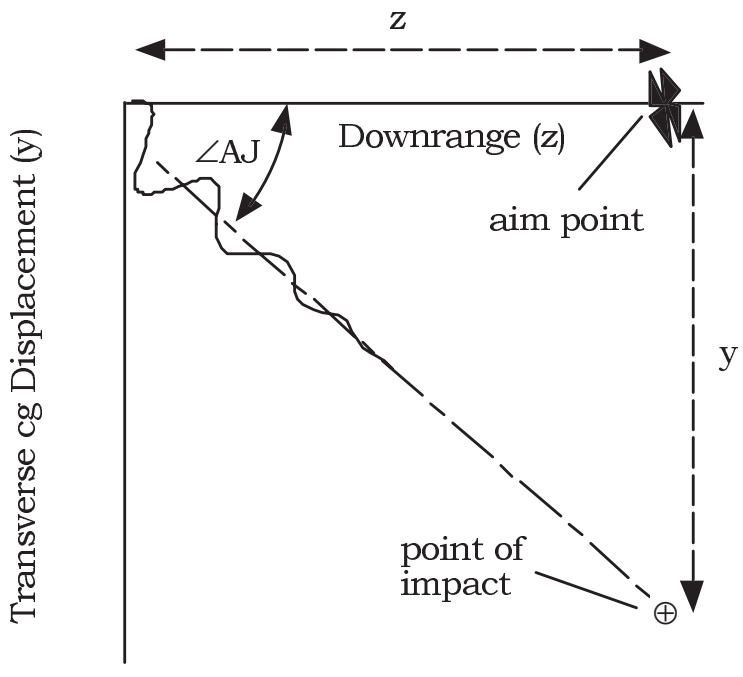

Fig. 4. Geometrical view of, neglecting.

Bundy [5], the axis of swerve symmetry runs parallel to the tangent to the swerve curve at any and all of the local swerve maxima (positive or negative maxima with respect to the swerve axis). Hence, $\angle A J$ can also be defined as the angular difference between the tangent to the $c g$ trajectory at the origin of FF and the tangent to the swerve curve at the first (or second, or third, etc.) local maximum in the swerving motion. In equation form, this alternative definition is stated as:

$$
\begin{aligned}
\angle A J= & \tan ^{-1}\left\{\left.\frac{d y}{d z}\right|_{z_{\text {swerve maxima }}}\right\} \\
& -\tan ^{-1}\left\{\left.\frac{d y}{d z}\right|_{\left.z_{\text {origin of free flight }}\right\}}\right. \\
\approx & \frac{d y}{d z}\left|z_{\text {swerve maxima }}-\frac{d y}{d z}\right|_{z_{\text {origin of free flight }}}
\end{aligned}
$$




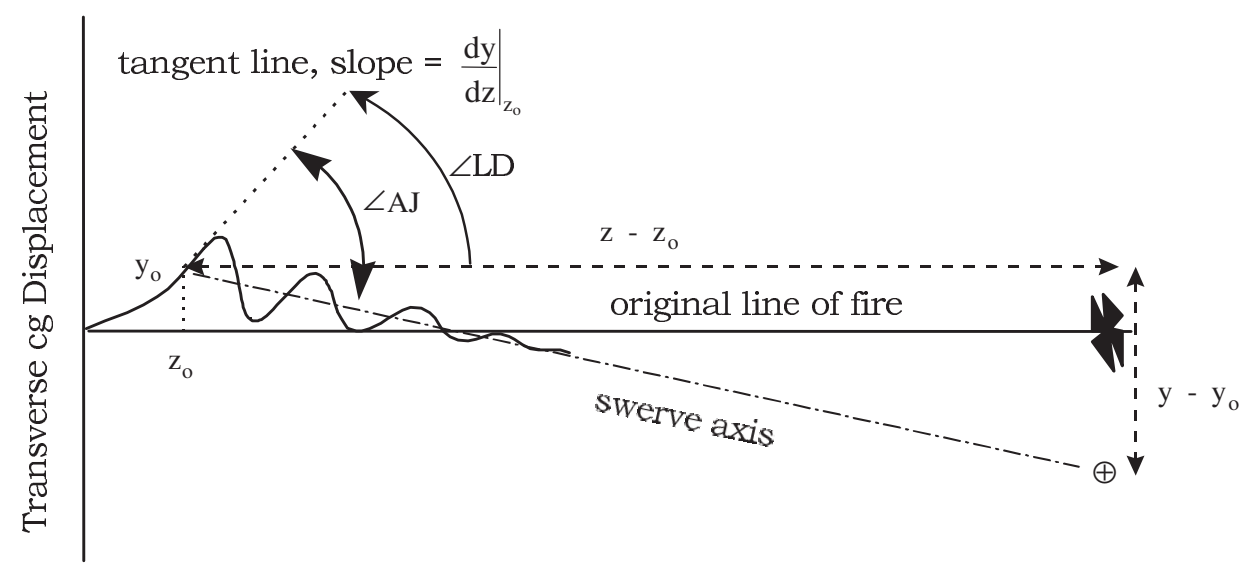

Fig. 5. Geometrical interpretation of Eq. (2).

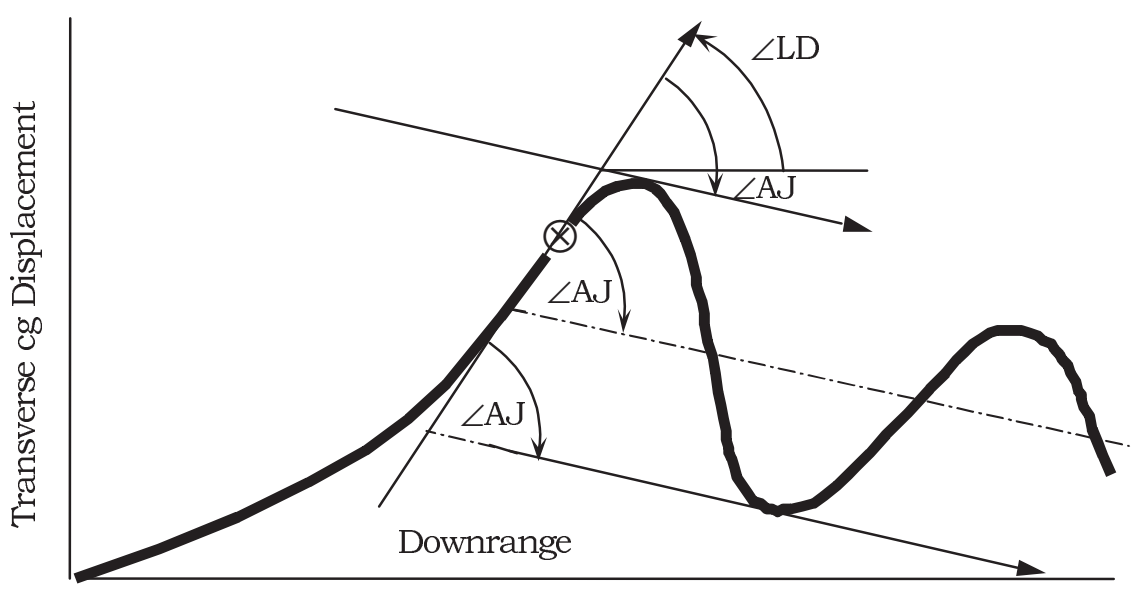

Fig. 6. Alternative geometrical definitions for.

where the subscripts identify the locations at which the derivatives are to be evaluated. Note, unlike Eqs (1) and (2), the definition for $\angle A J$ given in Eq. (3) does not call upon the limit as the trajectory approaches infinity.

Even though $\angle A J$ can be defined using the tangent line at any of the local maxima, as depicted in Fig. 6, it is clear that the minimum distance needed to establish the orientation of the swerve axis is the distance to the first swerve maxima, $z_{1}$. Thereafter, the $c g$ motion simply oscillates back and forth about this axis, albeit with damped amplitude.

The "limitless" definition of Eq. (3) facilitates additional insights into the kinematic relationship between $\angle A J$ and the initial conditions at the origin of FF. Take the special cases illustrated in Figs 7(a) and (b), unlike Fig. 6, the swerve axes in these two cases are nearly parallel with the LD direction, hence, $\angle A J$ is nearly zero. From Fig. 7(a), for instance, the distance between the origin of free fight, $z_{o}$, and the first swerve maximum, $z_{1}$, is relatively small, at least in comparison with the wavelength of the swerve curve, $\lambda$. On the other hand, in Fig. 7(b), $z_{1}-z_{0}$ is relatively large. Contrasting the larger $\angle A J$ of Fig. 6 with that of Figs 7(a) and (b), it can be inferred that the largest $\angle A J$ will occur when $z_{1}-z_{o}=\lambda / 4$. In fact, if the swerve curve is approximated by a sine wave of the form $y=A \sin (2 \pi[z-z-o] / \lambda)$, at least for the first cycle, then, from Eq. (3), the maximum $\angle A J$ would be given by Eq. (4).

To appreciate the significance of Eq. (4), Fig. 8 illustrates how $\angle A J_{\max }$ varies with $A$ and $\lambda$ for two cases where $y$ conforms to $A \sin \left(2 \pi\left[z-z_{o}\right] / \lambda\right)$. From the depiction, a larger $A$ and smaller $\lambda$ produce a larger $\angle A J_{\max }$. For large-caliber guns, $A$ may be on the order of several millimeters, whereas $\lambda$ is on the order of tens of meters; hence, $\angle A J_{\max }$, from Eq. (4), will be small - on the order of milliradians. 
a)

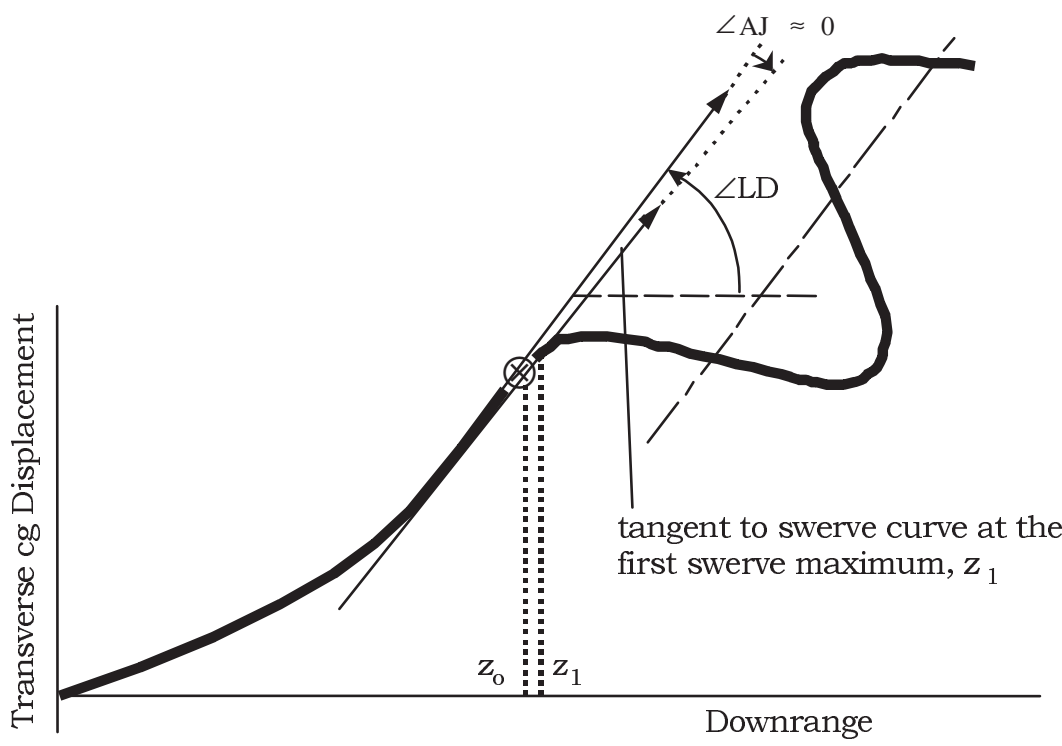

b)

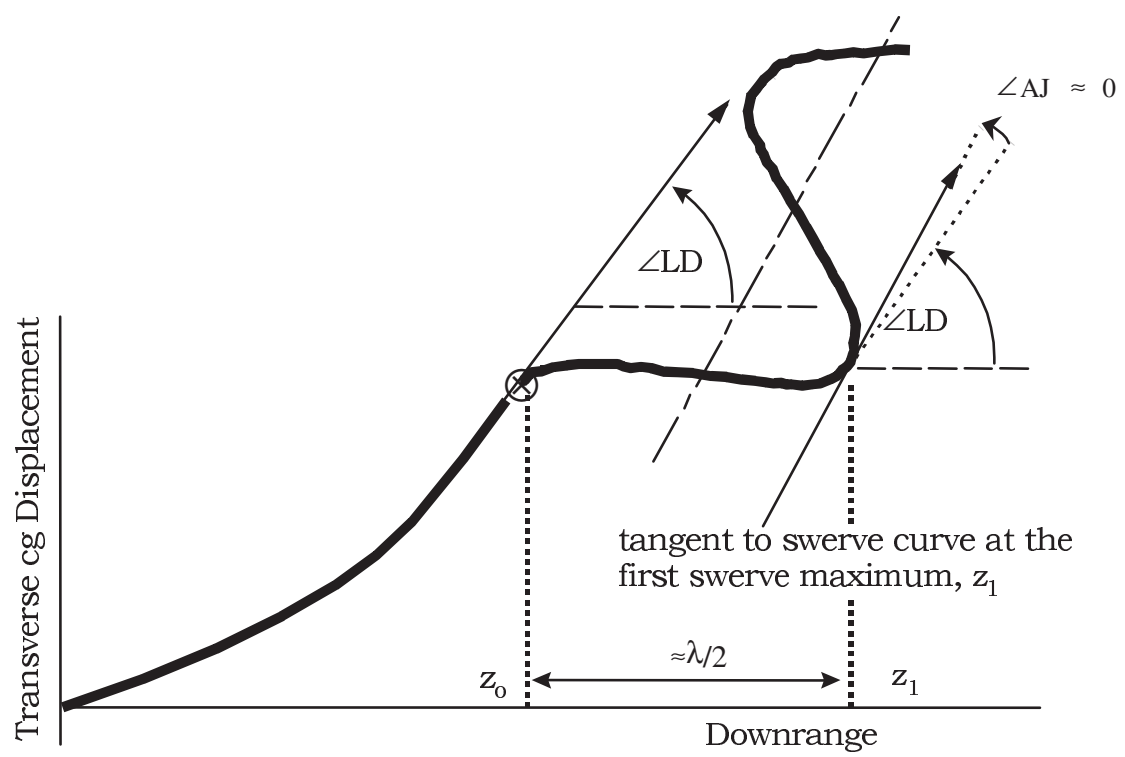

Fig. 7. The influence of initial (swerve) conditions on AJ, a) Entry into FF at $z_{0} \approx z_{1}$, and b) Entry into $\mathrm{FF}$ at $z_{0} \approx z_{1}-\lambda / 2$.

$$
\begin{aligned}
& \left|\angle A J_{\max }\right| \\
= & \left|\frac{d\left\{y=A \sin \left(\frac{2 \pi\left[z-z_{o}\right]}{\lambda}\right)\right\}}{d z}\right|_{z_{1}=z_{o}+\lambda / 4} \\
& -\frac{d\left\{y=A \sin \left(\frac{2 \pi\left[z-z_{o}\right]}{\lambda}\right)\right\}}{d z} \mid \\
= & \frac{A 2 \pi}{\lambda}
\end{aligned}
$$

In summary, Figs 6-8, and the discussions thereabout, illustrate that the axis of swerve symmetry is fixed in space by the time the rod reaches its first swerve maximum, as implied by Eq. (3). They also provide visible examples that support the contention that it is not necessary to take the swerving motion to infinity, as called for in Eqs (1) and (2), in order to establish the swerve axis. However, geometry alone does not provide physical insight into how the $c g$ path can turn away from the LD direction after the rod enters FF. Next, a physical model is constructed that identifies the aero- 


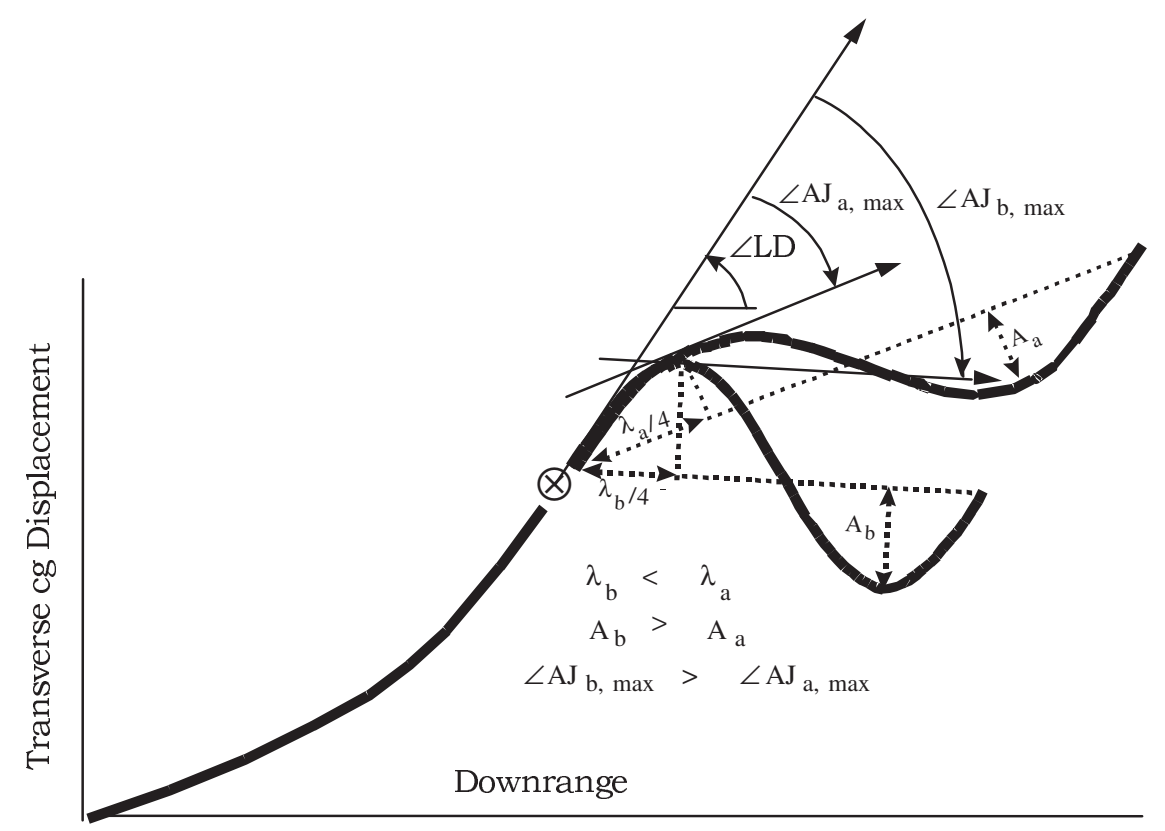

Fig. 8. Depiction of variation in (AJ max with amplitude and wavelength of the swerve curve.

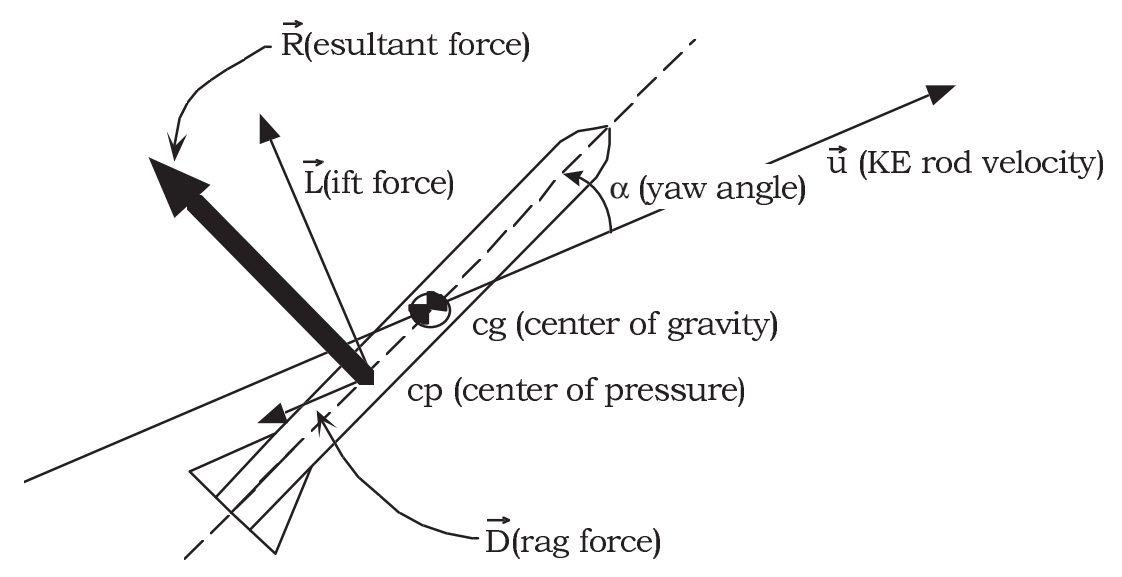

Fig. 9. Illustrating the lift and drag force directions, as well as the CG and CP locations.

dynamic force that causes such a change in direction to occur.

\section{A physical explanation for $\mathrm{AJ}$}

A KE penetrator is also called a long-rod penetrator, conveying the fact that its fundamental shape is a high length-to-diameter ratio cylinder. It is fin stabilized, with only a small roll rate (assumed here to be zero). There are two fundamental aerodynamic forces on such a rod, one is drag, $\vec{D}$, and the other is lift, $\vec{L}$. The combination of drag and lift produces a resultant force, $\vec{R}(=\vec{L}+\vec{D})$, acting at the center of pressure, cp, as shown in Fig. 9. The drag force is opposite in direction to, while the lift force is perpendicular to, $\vec{u}$. However, lift is only nonzero if the angle of yaw $(\alpha)$ between the symmetric rod axis and $\vec{u}$ is nonzero. Since the Magnus force is absent for a nonrolling projectile, lift is the only aerodynamic force that is capable of producing the type of lateral $c g$ motion needed to explain AJ.

In order to understand how the lift force can, in fact, account for AJ, it is necessary to discuss the factors that control $\alpha$ (thereby controlling lift). By Newton's 


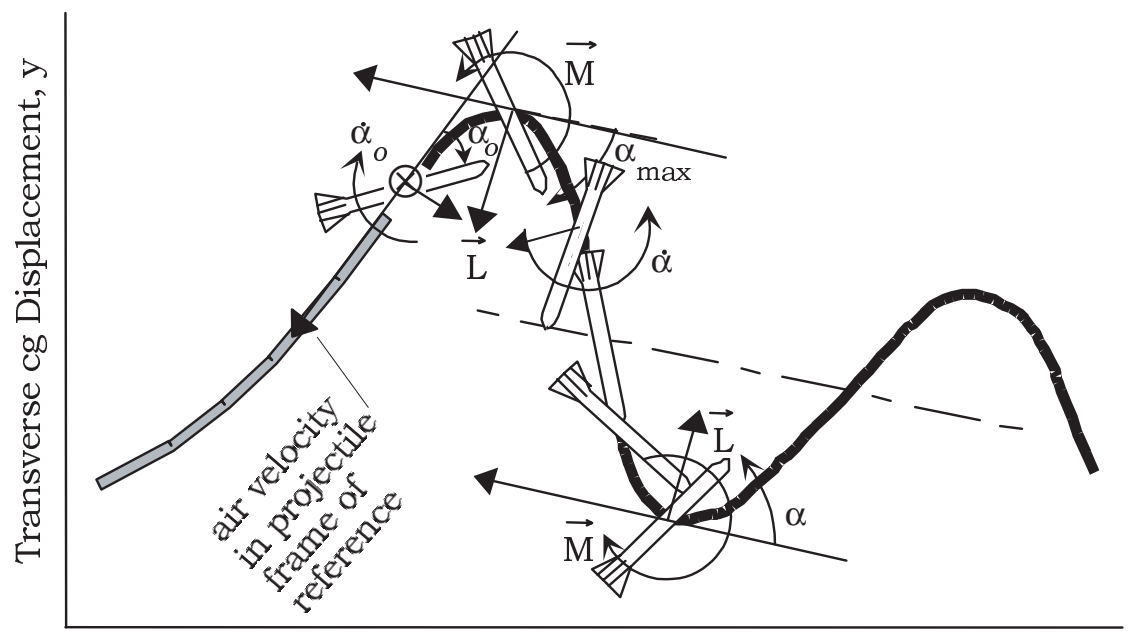

Downrange, $z$

Fig. 10. Angular orientations of a KE rod consistent with the CG trajectory of Figs 2, 3, 5 and 6.

second law for angular motion, yaw will be controlled by the aerodynamic moment, $\vec{M}$, that acts about the projectile $c g$. For a properly designed nonspinning KE rod, $\vec{M}$ will tend to decrease $\alpha$, aligning the rod axis with $\vec{u}$. Consider then, what happens when such a KE penetrator enters FF.

Suppose, as shown in Fig. 10 (which displays the same $c g$ motion as Figs 2, 3, 5, 6), the transverse component of the $c g$ velocity is positive at the point where the rod enters FF, i.e., $\dot{y}_{o}>0$ at $y_{0}, z_{0}$ (attributed to a particular combination of LD effects, e.g., Fig. 1). Furthermore, assume that at entry into FF, the KE rod has a negative initial yaw, $\alpha_{o}<0$ (represented, as a clockwise rotation of the rod axis below the impinging airstream in Fig. 10) and a negative initial yawing rate, $\dot{\alpha}_{o}<0$ (given a clockwise arrow). Under these conditions, the flow of air will create an initial lift force with a negative- $y$ component (reducing $\dot{y}$ ), and a negative- $x$ directed moment $\vec{M}$, which (in lieu of being out of the page in Fig. 10) is depicted as a counterclockwise arrow. Eventually, the negative lift force will turn the $\mathrm{cg}$ motion around, so that $\dot{y}<0$. Likewise, the counterclockwise $\vec{M}$ will eventually turn the angular motion around, changing the yaw rate from its initial negative value (clockwise) to a positive one (counterclockwise), $\dot{\alpha}>0$. These changes in direction occur around the first local maximum in the swerve curve. In fact, it can be shown (Bundy [5]) that the yaw angle will be at its maximum at the local swerve maximum.

After bringing the clockwise rotation to a halt at its maximum (negative) yaw angle, $\alpha_{\max }$, the counterclockwise moment in the first half cycle reverses the yaw rotation and eventually brings $\alpha$ to zero. This occurs when the y component of the rod's $c g$ crosses the swerve axis, as pictured. Throughout this period of time, the lift force remains negative; thus, the rod $c g$ will enter the second half cycle of the swerve motion with negative- $y$ momentum. Similarly, the counterclockwise angular momentum rotates $\alpha$ past zero at the swerve axis, giving the rod a positive yaw in the second half cycle. However, when $\alpha$ becomes positive, it generates a positive lift force and a positive- $x$ directed (into the page) clockwise moment $\vec{M}$ that opposes further increases in $\alpha$, as depicted in Fig. 10. Such projectiles are said to be statically stable, which simply means that if the projectile is held statically at some nonzero yaw angle, and then released (as might be the case in a wind tunnel experiment), the yaw will decrease.

Hence, the conditions present in the first half cycle of FF are exactly reversed in the second half of the swerve cycle. Therefore, with the exception of a reversal in directions, the explanation for events in the second half cycle will be identical to that which accounted for the first half cycle motion. In a like manner, each cycle in the swerving motion of the KE rod $c g$ can be understood as resulting from the oscillation in lift created by a periodic motion in yaw.

Although the previous arguments infer that the change in $c g$ direction between the point of entry into $\mathrm{FF}$ and the first maximum yaw ( $\equiv \angle A J)$ is attributable to the force of lift due to yaw over this region, the dependence of $\angle A J$ on initial conditions cannot be fully appreciated until Fig. 7 is compared with Fig. 6 in the following manner. 


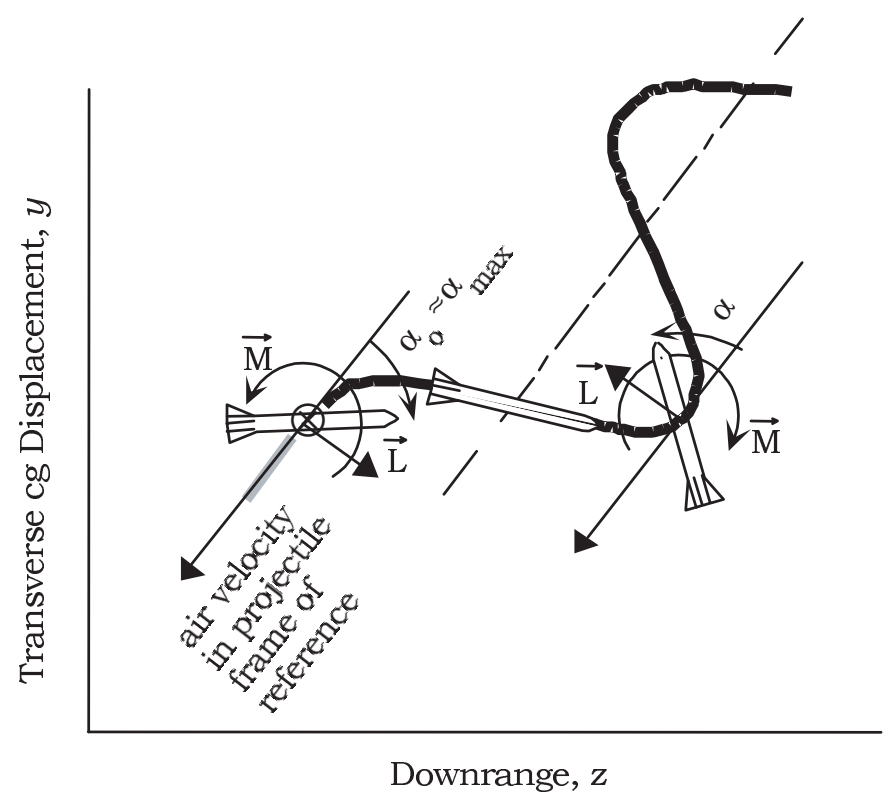

Fig. 11. Angular orientations of a KE rod consistent with the CG trajectory of Fig. 7.

Figure 11, displays a set of initial and subsequent FF conditions that are consistent with the $c g$ motion of Fig. 7. Specifically, as illustrated, the initial yaw angle of the rod as it enters FF is near its maximum (albeit negative) value, $\alpha_{o} \approx \alpha_{\max }$, therefore, the initial yaw rate is near zero, $\dot{\alpha}_{o} \approx 0$. Furthermore, since the initial yaw is negative, $\vec{L}$ is initially directed toward the swerve axis and $\vec{M}$ is initially in the negative- $x$ direction (out of the page). These are exactly the same conditions that were present at the first local maximum in the swerving motion of Fig. 10. Therefore, from the point of first maximum yaw onward, the lift force affecting the $\mathrm{cg}$ motion of Fig. 11 is exactly the same as that governing the $c g$ motion of Fig. 10. Yet, the direction of the swerve axis is seen to be noticeably different, in particular, the $\angle A J$ is noticeably different. The significance of this observation is the sum and substance of this paper. That is, since the aerodynamic force on the KE rod is essentially the same from the first maximum onward in both Figs 10 and 11 , yet $\angle A J$ is significantly different in the two figures, it must be the case that $\angle A J$ is due to differences in the lift force prior to the first maximum yaw. Once again, implicating that $\mathrm{AJ}$ is a regional effect, occurring from the point of entry into FF up to the point of first maximum yaw.

Ultimately, differences in the lift force, more correctly, differences in the integrated lift force, up to the point of first maximum yaw are governed by differences in the initial conditions. That is, in both Figs 10 and 11 the rod enters FF with an appreciable initial yaw angle, but only in the case of Fig. 10 was the initial yaw rate, $\dot{\alpha}_{o}$, notably different than zero. Comparing this with the observation that $\angle A J$ was only substantial for the initial conditions of Fig. 10, it can be deduced that $\angle A J$ is not dependent upon the initial yaw angle, but rather, it is dependent on the initial yaw rate. It is shown later that, indeed, the mathematical expression for $\angle A J$ of a nonspinning $\mathrm{KE}$ rod depends on the initial yaw rate, not yaw. (Bear in mind, for the more general case of a spinning projectile, $\angle A J$ depends on both yaw and yaw rate.)

In summary, based on the physical and kinematical developments of this and the previous section, respectively, it is now a simple matter to derive a dynamical expression for AJ. However, before such an expression can be formulated, it is necessary to review the basic aerodynamic forces and moments.

\section{Basic aerodynamic forces and moments acting on a nonspinning ke penetrator}

The force of friction, drag, on the projectile is commonly expressed as

$$
\vec{D}=-\frac{1}{2} C_{D} \rho A|\vec{u}| \vec{u}
$$

where $C_{D}$ is called the drag coefficient, $\rho$ is the air density, $A$ is the cross-sectional area of the projectile, and, by virtue of the minus sign, drag is in the direction 


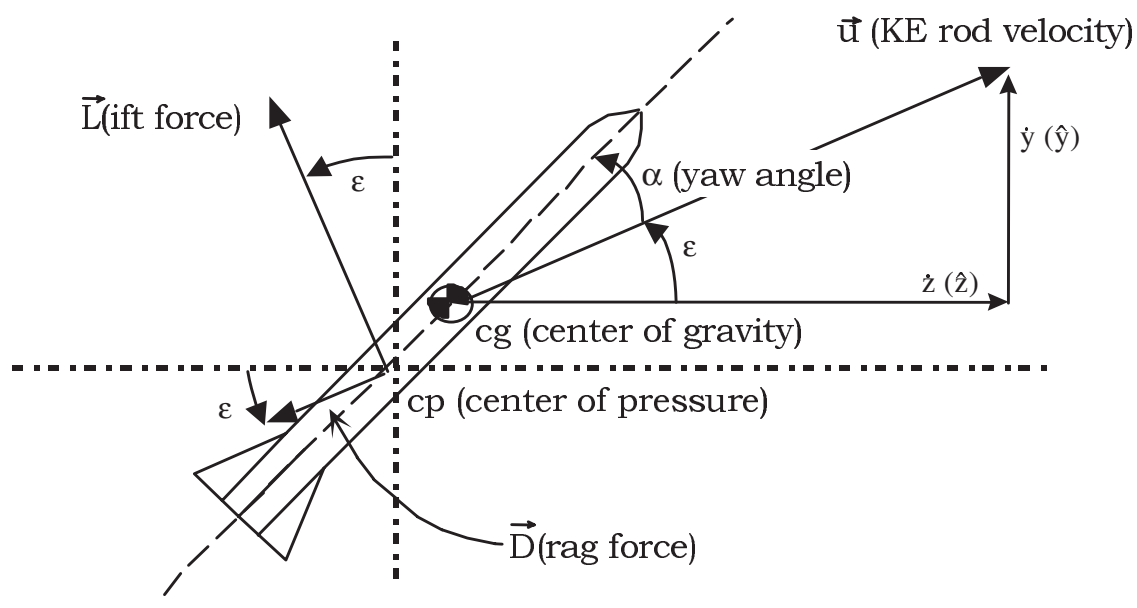

Fig. 12. Illustration of 2-D planar force and velocity components.

opposite $\vec{u}$, the $c g$ velocity vector (Fig. 9). For small yaw (e.g., $\alpha<5^{\circ}$ ), typical of KE rods, $C_{D}$ can be considered a constant.

The expression for lift is conventionally written as

$$
\vec{L}=\frac{1}{2} C_{L} \rho A|\vec{u}|^{2} \hat{L},
$$

where $C_{L}$ is referred to as the lift coefficient. The unitvector direction of the lift force, $\hat{L}$, is perpendicular to the drag force and is in the yaw plane.

Here, yaw is the vertical $(z-y)$-plane angle, $\alpha$, between the projectile's tail-to-nose axis and the tangent to its trajectory (or, equally suitable, $\vec{u}$ ). As is typical, it is assumed here that a positive $\alpha$ means the nose of projectile is above $\vec{u}$. A projectile flying at zero yaw has no lift, whereas one having a positive $\alpha$ generates a positive lift (the case depicted in Fig. 9) and one having a negative $\alpha$ creates a negative lift (the initial conditions depicted in Figs 10 and 11). A yaw dependence of this type is characteristic of an odd-power series in $\alpha$. For small yaw, the expansion can be truncated after the first power in $\alpha$ :

$$
C_{L}=C_{l_{\alpha}} \alpha
$$

where $C_{l_{\alpha}}$ is the derivative of the lift coefficient with respect to $\alpha$.

Suppose the original line of fire is defined to be the $z$ axis, and that gravity and the Coriolis force are ignored. Furthermore, assume the $c g$ motion is 2-D planar, in particular, assume the motion is confined to the vertical plane, then $\vec{u}=\dot{z} \hat{z}+\dot{y} \hat{y}$ (where a dot indicates time differentiation), as shown in Fig. 12.

From Newton's second law for linear motion in the $\hat{y}$ direction,

$$
\begin{aligned}
m \frac{d \dot{y}}{d t} & =\vec{L} \cdot \hat{y}+\vec{D} \cdot \hat{y} \\
& =\frac{\alpha}{|\alpha|}\{|\vec{L}| \cos \varepsilon\}-|\vec{D}| \sin \varepsilon,
\end{aligned}
$$

where $m$ is the mass of the projectile, and the ratio $\alpha /|\alpha|$ accounts for the positive or negative influence of yaw on lift. The counterclockwise (positive) angular deviation, $\varepsilon$, of $\vec{u}$ from the original line of fire is assumed small; nevertheless, it is not necessary to neglect $\varepsilon$ entirely in order to simplify Eq. (8). If the coordinate axes $\hat{z}$ and $\hat{y}$ are simply rotated by the angle $\varepsilon$ (i.e., the $\tan ^{-1}$ of the $c g$ trajectory) at $z_{1}$ and $y_{1}$, and thereafter denoted $\hat{s}$ and $\hat{Y}$, respectively, as shown in Fig. 13, then the equation of motion in the $\hat{Y}$ direction becomes

$$
m \frac{d \dot{Y}(s)}{d t}=\vec{L} \cdot \hat{Y}+\vec{D} \cdot \hat{Y} \approx \frac{\alpha}{|\alpha|}|\vec{L}|,
$$

where lift is nearly parallel with, and drag nearly perpendicular to, the $Y$ axis (even though it may not appear this way in the not-to-scale illustration of Fig. 13.). In this coordinate system, the locus of points $Y(s)$ defines the swerve curve, which oscillates about the swerve axis, $\hat{s}$. Note, $\vec{u}$ will actually oscillate about some average vector; however, the magnitude of this oscillation is small, so that, $\vec{u}(=\dot{z} \hat{z}+\dot{y} \hat{y}) \approx \dot{s} \hat{s}$.

(Note also, for future reference, if the direction of positive $\hat{y}$ and positive $\hat{Y}$ had been defined as opposite of that assigned here [viz., if $\hat{y}$ and $\hat{Y}$ were defined as positive downward], yet $\alpha$ was still considered positive when the nose of the KE rod is above its $c g$ velocity vector, then the right-hand side of Eqs (8) and (9) would change signs. This minus sign would carry through in subsequent equations; ultimately, it would change the sign of the final expression for AJ. In other words, 


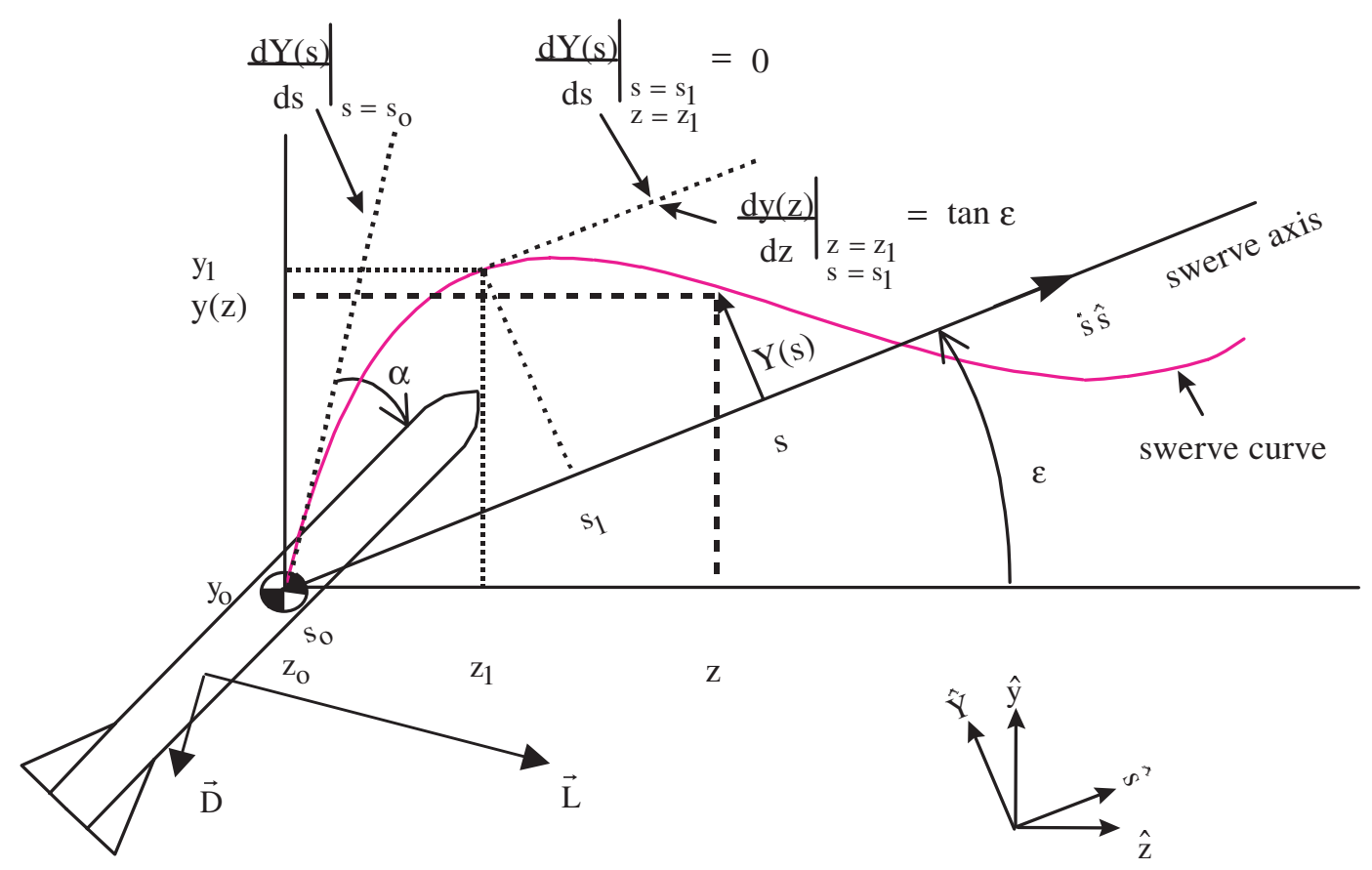

Fig. 13. Swerve-fixed $(\hat{s}, \hat{Y})$ and earth-fixed $(\hat{z}, \hat{y})$ coordinate system (illustrated dimensions are not to scale).

as stated in the kinematical discussion of $\mathrm{AJ}$, the sign convention for positive transverse displacement will affect the sign convention for AJ.)

Simply stated, Eq. (9) establishes that lift is the primary cause of swerve, and, from Eqs (6) and 7, lift is proportional to yaw; hence

$$
\begin{aligned}
m \frac{d \dot{Y}(s)}{d t} & =\left.\frac{\alpha}{|\alpha|}\left|\frac{1}{2} \rho A\right| \vec{u}\right|^{2} C_{l_{\alpha}} \alpha \mid \\
& =\frac{1}{2} \rho A|\vec{u}|^{2} C_{l_{\alpha}} \alpha .
\end{aligned}
$$

The expression for $\alpha$ must satisfy the torque equation, viz.,

$$
m k^{2} \frac{d \dot{\alpha}}{d t}(-\hat{x})=\vec{M}
$$

where $k^{2}$ is the radius of gyration of the KE rod about its transverse $(x)$ axis. Furthermore, in accordance with the right-hand rule, a counterclockwise angular acceleration of the rod (relative to the positive-s(werve) axis) must be represented by a negative- $x$ directed vector (which points out of the page), hence, the negative unit vector, $-\hat{x}$, appears in Eq. (11).

In FF, the axis of the projectile will oscillate about its $c g$ trajectory (i.e., the $z, y$ or $s, Y$ curve, depending on the coordinate system chosen). Just as air opposes the forward motion of the projectile, it will also oppose this oscillating motion. Hence, there will be a resist- ing torque, known as the damping moment, that varies with the yaw rate. As the name implies, the damping moment causes the yaw magnitude to diminish with time of flight. However, since it has been argued in the previous illustrations (e.g., Figs 6-8) that $\angle A J$ is established within a relatively short segment of the trajectory, the effect of damping on AJ can be ignored. In this case, the moment $\vec{M}$ about the $c g$ will only be due to the resultant force, $\vec{R}=\vec{L}+\vec{D}$, located at the center of pressure, $c p$ (Fig. 12). Thus,

$$
\begin{aligned}
\vec{M}= & |c g-c p| \\
& \left(|\vec{D}| \sin \alpha+\frac{\alpha}{|\alpha|}|\vec{L}| \cos \alpha\right) \hat{x} \\
& \approx|c g-c p|\left(|\vec{D}| \alpha+\frac{\alpha}{|\alpha|}|\vec{L}|\right) \hat{x}
\end{aligned}
$$

for small $\alpha\left(\right.$ e.g., $\left.<5^{\circ}\right)$. Note, a positive $\alpha$ will generate a positive- $x$ directed $\vec{M}$.

Using Eqs (5)-(7) in Eq. (12), yields

$$
\begin{aligned}
\vec{M} & =\frac{1}{2} \rho A|\vec{u}|^{2}|c g-c p| \alpha\left(C_{D}+C_{l_{\alpha}}\right) \hat{x} \\
& =-\frac{1}{2} C_{m_{\alpha}} \rho A|\vec{u}|^{2} d \alpha \hat{x},
\end{aligned}
$$

where $C_{m_{\alpha}}\left(=-\left[C_{D}+C_{l_{\alpha}}|c g-c p| / d\right)\right.$ is called the derivative of the restoring (overturning, or pitching) moment coefficient with respect to $\alpha$, and $d$ is the rod 
diameter. By definition, $C_{m_{\alpha}}$ is negative for a statically stable projectile. The aerodynamic coefficients $C_{D}$, $C_{l_{\alpha}}$, and $C_{m_{\alpha}}$ can best be determined by free flight tests conducted in an aeroballistic range, such as the US Army Research Laboratory's Transonic Range.

Substituting Eq. (13) into Eq. (11) produces

$$
m k^{2} \frac{d \dot{\alpha}}{d t}=\frac{1}{2} C_{m_{\alpha}} \rho A|\vec{u}|^{2} d \alpha .
$$

Since $C_{m_{\alpha}}$ is negative for the KE projectile, this differential equation for $\alpha$ is of the form $\ddot{\alpha} \propto-\alpha$. Such an equation has a sinusoidal solution; thus, from Eq. (10), $Y(s)$ will have a sinusoidal solution (however, $\alpha$ and $Y$ will be $180^{\circ}$ out of phase). It is now proven that this oscillatory motion, coupled with the lift force, can account for AJ.

\section{A mathematical formulation for $\mathbf{A J}$}

It was stated in the kinematical discussion surrounding Eq. (3), that an alternative definition for $\angle A J$ is:

$$
\begin{aligned}
\angle A J & =\tan ^{-1}\left\{\left.\frac{d y}{d z}\right|_{z_{1}}\right\}-\tan ^{-1}\left\{\left.\frac{d y}{d z}\right|_{z_{o}}\right\} \\
& \left.\approx \frac{d y}{d z}\right|_{z_{1}}-\left.\frac{d y}{d z}\right|_{z_{o}} \\
( & \left.=\left.\frac{\dot{y}}{\dot{z}}\right|_{z_{1}}-\left.\frac{\dot{y}}{\dot{z}}\right|_{z_{o}} \approx \frac{\dot{y}\left(z_{1}\right)-\dot{y}\left(z_{o}\right)}{\dot{z}}\right)
\end{aligned}
$$

where, it will be recalled, $z_{1}$ is taken to be the downrange coordinate of the first local swerve maxima (relative to the swerve axis), while $z_{o}$ is the designation for the downrange coordinate at the origin of FF (c.f., Figs 7 and 13). Hence, from Eq. (15), $\angle A J$ can be viewed as a change in slope of the cg trajectory from $z_{o}$ to $z_{1}$ (or, it can be viewed as a change in transverse velocity from $z_{o}$ to $z_{1}$, nondimensionalized by the longitudinal velocity [assumed to be constant from $z_{o}$ to $\left.\left.z_{1}\right]\right)$.

Equations 3 and 15 define $\angle A J$ in terms of $d y / d z$, to find its equivalent expression in terms of $d Y / d s$, it is necessary to find the transformation algorithm between $y$ and $Y$, and $z$ and $s$. To that end (with the aid of Fig. (13)), it can be shown that

$$
\begin{aligned}
& y(z)=Y(s) \cos \varepsilon+\left(s-s_{o}\right) \sin \varepsilon \\
& z=\left(s-s_{o}\right) \cos \varepsilon-Y(s) \sin \varepsilon
\end{aligned}
$$

From Eq. (16),

$$
\begin{aligned}
\frac{d y(z)}{d z} & =\frac{\frac{d y(z)}{d s}}{\frac{d z}{d s}}=\frac{\frac{d Y(s)}{d s} \cos \varepsilon+\sin \varepsilon}{\cos \varepsilon-\frac{d Y(s)}{d s} \sin \varepsilon} \\
& \approx \frac{d Y(s)}{d s}+\tan \varepsilon, \text { for } \frac{d Y(s)}{d s}, \varepsilon \ll 1
\end{aligned}
$$

Hence, from Eqs (15) and (17),

$$
\begin{aligned}
\angle A J & =\left.\frac{d y}{d z}\right|_{z_{1}, s_{1}}-\left.\frac{d y}{d z}\right|_{z_{o}, s_{o}} \\
& =\left.\frac{d Y}{d s}\right|_{s_{1}, z_{1}}-\left.\frac{d Y}{d s}\right|_{s_{o}, z_{o}} \\
& \left(=\left.\frac{\dot{Y}}{\dot{s}}\right|_{s_{1}}-\left.\frac{\dot{Y}}{\dot{s}}\right|_{s_{o}} \approx \frac{\dot{Y}\left(s_{1}\right)-\dot{Y}\left(s_{o}\right)}{|\vec{u}|}\right),
\end{aligned}
$$

where the subscript notation $z_{1}, s_{1}$ (for example) refers to the point on the swerve curve with coordinate $z_{1}$ along the $z$ axis and $s_{1}$ along the $s$ axis (c.f. Fig. 13), and time and space derivatives are related by

$$
\dot{Y}=\frac{d Y}{d t}=\frac{d Y}{d s} \frac{d s}{d t} \approx|\vec{u}| \frac{d Y}{d s} .
$$

In effect, Eq. (18) states the obvious - the difference in slopes between two points on the swerve curve does not change if the coordinate system, used to describe the curve, is rotated through an angle $\varepsilon$.

Combining Eqs (9), (10) and (14), it can be shown that

$$
d \dot{Y}=\frac{(\vec{L} \cdot \hat{Y}) d t}{m}=\frac{C_{l_{\alpha}} k^{2}}{d C_{m_{\alpha}}} d \dot{\alpha} .
$$

Denoting $\dot{\alpha}_{o}$ and $s_{o}$ as the initial conditions at entry into FF and $\dot{\alpha}_{1}$ and $s_{1}$ as the conditions at the first local maximum in the swerve curve, then integration of Eq. (20) yields

$$
\begin{aligned}
\dot{Y}\left(s_{1}\right)-\dot{Y}\left(s_{o}\right) & =\int_{\dot{Y}\left(s_{o}\right)}^{\dot{Y}\left(s_{1}\right)} d \dot{Y} \\
& =\frac{1}{m} \int_{t\left(s_{o}\right)}^{t\left(s_{1}\right)}(\vec{L} \cdot \hat{Y}) d t \\
& =\int_{\dot{\alpha}_{o}}^{\dot{\alpha}_{1}} \frac{C_{l_{\alpha}} k^{2}}{d C_{m_{\alpha}}} d \dot{\alpha} \\
& =\frac{C_{l_{\alpha}} k^{2}}{d C_{m_{\alpha}}}\left(\dot{\alpha}_{1}-\dot{\alpha}_{o}\right) .
\end{aligned}
$$

Combining Eqs (18) and (21), yields

$$
\angle A J=\left.\frac{d Y}{d s}\right|_{s_{1}, z_{1}}-\left.\frac{d Y}{d s}\right|_{s_{o}, z_{o}}
$$




$$
\begin{aligned}
& =\frac{1}{m|\vec{u}|} \int_{t\left(s_{o}\right)}^{t\left(s_{1}\right)}(\vec{L} \cdot \hat{Y}) d t \\
& =\frac{C_{l_{\alpha}} k^{2}}{d C_{m_{\alpha}}|\vec{u}|}\left(\dot{\alpha}_{1}-\dot{\alpha}_{o}\right) .
\end{aligned}
$$

Equation (22) shows that $\angle A J$ can be viewed as a change in the slope of the cg trajectory from $z_{o}$ to $z_{1}$, or, it can be related to a change in angular rates from $z_{o}$ to $z_{1}\left(s_{o}\right.$ to $\left.s_{1}\right)$. Furthermore, the insertion of the lift correlation in Eq. (20) and its retention in Eqs (21) and (22) serves to underscore the physical explanation given earlier, viz., that $\angle A J$ is due to the (integrated) effect of lift, caused by yaw, from $z_{o}\left(s_{o}\right)$ to $z_{1}\left(s_{1}\right)$.

Note, Eqs (18), (21) and (22) could have been simplified by setting $d Y /\left.d s\right|_{s_{1}, z_{1}}=0$ and $\dot{Y}\left(s_{1}\right)=0$, since, by definition, $Y(s)$ is at a local maxima at $s_{1}, z_{1}$. (This would also mean [from Eq. (17)] that $d y /\left.d z\right|_{z_{1}, s_{1}}=\tan \varepsilon$, as marked in Fig. 13.) Moreover, since $\alpha$ and $Y$ are $180^{\circ}$ out of phase, when $\dot{Y}\left(s_{1}\right)=0$, $\dot{\alpha}_{1}=0$; hence, Eq. (22) can be simplified to:

$$
\angle A J=-\left.\frac{d Y}{d s}\right|_{s_{o}, z_{o}}=-\frac{C_{l_{\alpha}} k^{2}}{d C_{m_{\alpha}}|\vec{u}|} \dot{\alpha}_{o} .
$$

From Eq. (22), it can be seen that $\angle A J$ will increase if either the integrand (viz., the lift force) or the domain of integration (viz. the lift force action time) increases. In more fundamental terms (noting that $C_{m_{\alpha}}$ will always be negative for a KE rod, and, $k, d$, and $|\vec{u}|$ are all positive), $\angle A J$ will increase if either 1) $C_{l_{\alpha}}$ increases (so that the lifting force per degree yaw increases), 2) $k$ increases (in which case, the rod would rotate slower, and hence the lifting force would act longer), 3) $\dot{\alpha}_{o}$ increases (so that, once again, it would take more time to bring the rod to rest), or 4) $C_{m_{\alpha}}$ decreases (so that the overturning moment per degree yaw decreases, again lengthening the action time for the lifting force).

Other, equivalent expressions for $\mathrm{AJ}$ that can be found in the literature include:

$$
\begin{aligned}
\angle A J & =-(+) \frac{C_{l_{\alpha}} k^{2}}{d C_{m_{\alpha}}|\vec{u}|} \dot{\alpha}_{o} \\
& \equiv-(+) \frac{C_{l_{\alpha}} I_{t}}{m|\vec{u}| d C_{m_{\alpha}}} \dot{\alpha}_{o} \\
& \equiv-(+) \frac{C_{l_{\alpha}} I_{t}}{m d^{2} C_{m_{\alpha}}} \alpha_{o}^{\prime},
\end{aligned}
$$

where $I_{t}\left(=m k^{2}\right)$ is the moment of inertia of the (symmetric) projectile about its transverse axis, and $\alpha_{o}^{\prime}$ is the initial FF yaw rate with respect to the trajectory arc length, measured in rod diameters (i.e., $\left.\alpha_{o}^{\prime} \equiv d \alpha /\left.d[s / d]\right|_{s_{o}, z_{o}}\right)$.
Depending on the coordinate system used, there may or may not be a negative sign on the right-hand side in the equalities/identities of Eq. (24). The convention chosen here is to define both the positive vertical axis $(y)$ and positive yaw $(\alpha)$ as up (up for $\alpha$ means its nose is above the cg velocity vector). However, it is more common in the field ballistics to define the positive vertical axis as down, and positive yaw as up; in this case, the negative sign convention is absent in Eq. (24) (thereby explaining the plus signs in parentheses). The plus-sign form of the expression for $\angle A J$ is, by far, the most common construction (e.g., Murphy and Bradley [3], Murphy [4], Fansler and Schmidt [6], Schmidt [7]). There is one other sign variation that may appear in the literature; if both the positive vertical axis and positive yaw are defined as down, then the sign remains negative in Eq. (24) (e.g., Murphy [2], Lijewski [8]). Regardless of the sign convention for the coordinate system used, it is always the case, as Murphy and Bradley [3] state, that "jump due to is in the direction of".

\section{Conclusions and commentary}

Equation (3) (or (15)) provides a "limitless" kinematical definition for $\angle A J$, which, reassuringly, leads to the traditional dynamical expression for $\angle A J$, viz. Eq. (24). The origins of possible variations in the sign convention of Eq. (24) were explored, but the paper's primary objective was to answer the questions: what is $\mathrm{AJ}$, what does it mean, and what aspect of the flight trajectory does it refer to, or account for.

For instance, one misconception about AJ can arise from the fact that Eq. (24) only shows a dependence on the initial yaw rate at the origin of FF (concealing the fact that it is actually a difference in rates, Eq. (22), that happens to equal the initial rate, Eq. (23)). Therefore, some may conclude from this (apparent) point dependence that $\mathrm{AJ}$ is a "point-based" phenomenon, i.e., that it results from (aero)dynamical effects that occur at the origin of FF. Others, seeking a geometrical explanation for AJ, may forgo the dynamical definition of Eq. (24) and return to its origin in the kinematical definition adopted, for example, by Murphy [2] or [4]. However, those geometry-based definition for AJ (viz., Eq. (1) or (2)) call for the cg coordinates to be evaluated in the limit of an infinite trajectory. Thus, there is some risk that those drawing upon this definition to explain AJ will erroneously assume that it is a transformation that accumulates with downrange distance 
(not realizing that the swerve axis is actually a constant, established long before the trajectory reaches infinity).

The central theme of this paper is to show that $\mathrm{AJ}$ is neither a change in direction that takes place at a point, nor is it a curving change that takes place over a domain of infinite extent, but rather, it is a regional transformation. In particular, using an alternative kinematic definition, it was illustrated geometrically (in terms of the cg trajectory), argued physically (in terms of yaw and lift), and proven mathematically (based on Newton's equations of motion), that $\angle A J$ for a (nonspinning) $\mathrm{KE}$ penetrator can be accounted for by the change in transverse cg velocity - due to lift - acting for the short period of time and space from entry of the projectile into FF until it reaches its first local maxima in yaw (or swerve).

\section{References}

[1] J. Bornstein, I. Celmins, P. Plostins and E.M. Schmidt, Techniques for the Measurement of Tank Cannon Jump, BRLMR-3715, US Army Ballistic Research Laboratory, Aberdeen
Proving Ground, MD, December 1988

[2] C.H. Murphy, Comments on Projectile Jump, BRL-MR-1071, US Army Ballistic Research Laboratories, Aberdeen Proving Ground, MD, AD 132323, April 1957.

[3] C.H. Murphy and J.W. Bradley, Jump Due to Aerodynamic Asymmetry of a Missile With Varying Roll Rate, BRL-R-1077, US Army Ballistic Research Laboratories, Aberdeen Proving Ground, MD, AD 219312, May 1959.

[4] C.H. Murphy, Free Flight Motion of Symmetric Missiles, BRLR-1216, US Army Ballistic Research Laboratories, Aberdeen Proving Ground, MD, July 1963.

[5] M.L. Bundy, The Regional Nature of Aerodynamic Jump, to be published, US Army Research Laboratory, Aberdeen Proving Ground, MD, December 1998.

[6] K.S. Fansler and E.M. Schmidt, The Influence of Muzzle Gasdynamics Upon the Trajectory of Fin-Stabilized Projectiles, BRL-R-1793, US Army Ballistic Research Laboratory, Aberdeen Proving Ground, MD, June 1975.

[7] E.M. Schmidt, A Technique for Reduction of Launch-Induced Perturbations, Proceedings of the Eighth US Army Symposium on Gun Dynamics, G.A. Pflegl, ed., Benet Special Publication ARCCB-SP-96032, Newport, RI, 14-16 May 1996, pp. 12-112-7.

[8] L.E. Lijewski, Aerodynamic Jump Prediction for Supersonic, High Fineness Ratio, Cruciform Finned Bodies, Journal of Guidance, American Institute of Aeronautics and Astronautics 5(5) (September-October 1982), 521-528. 

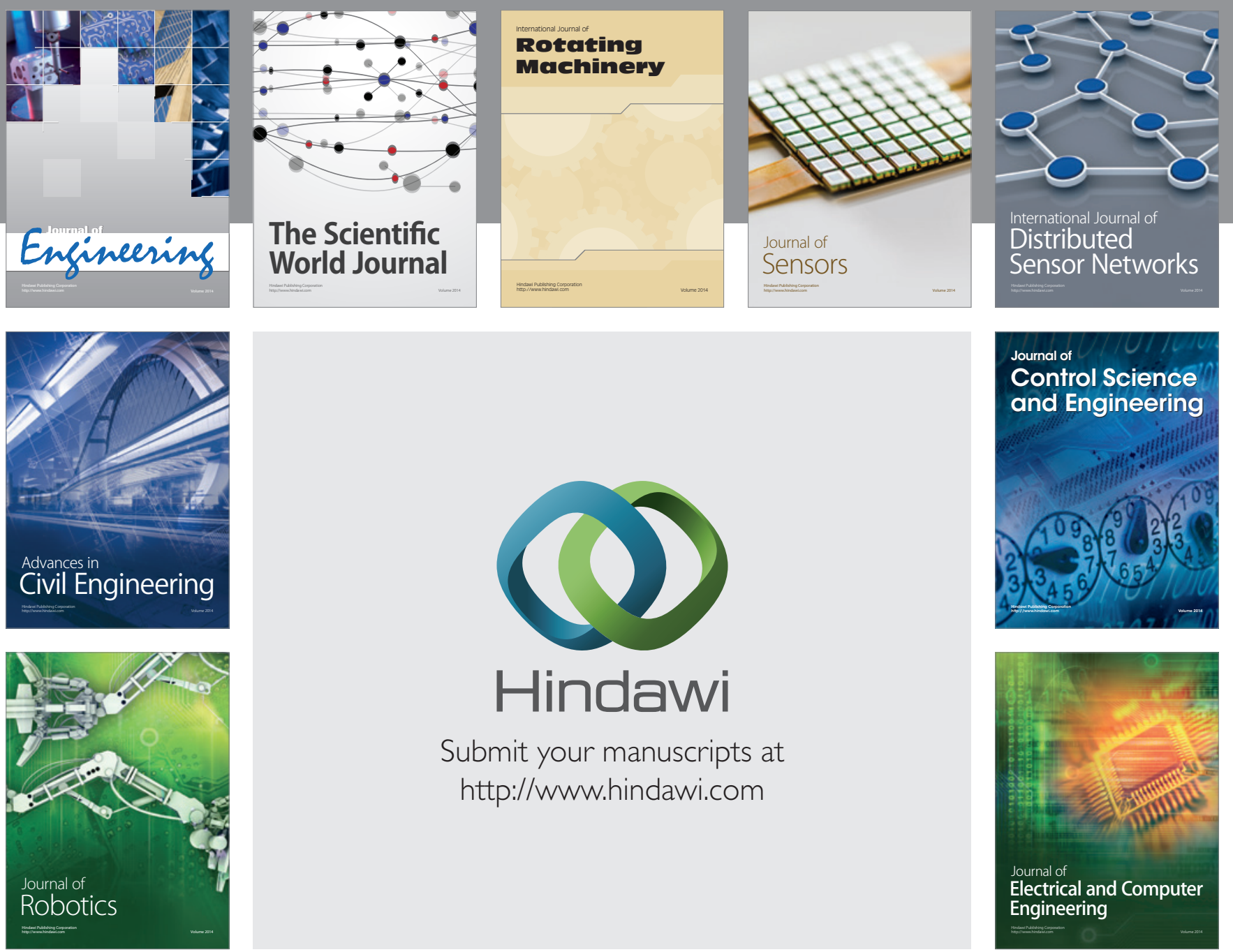

Submit your manuscripts at

http://www.hindawi.com
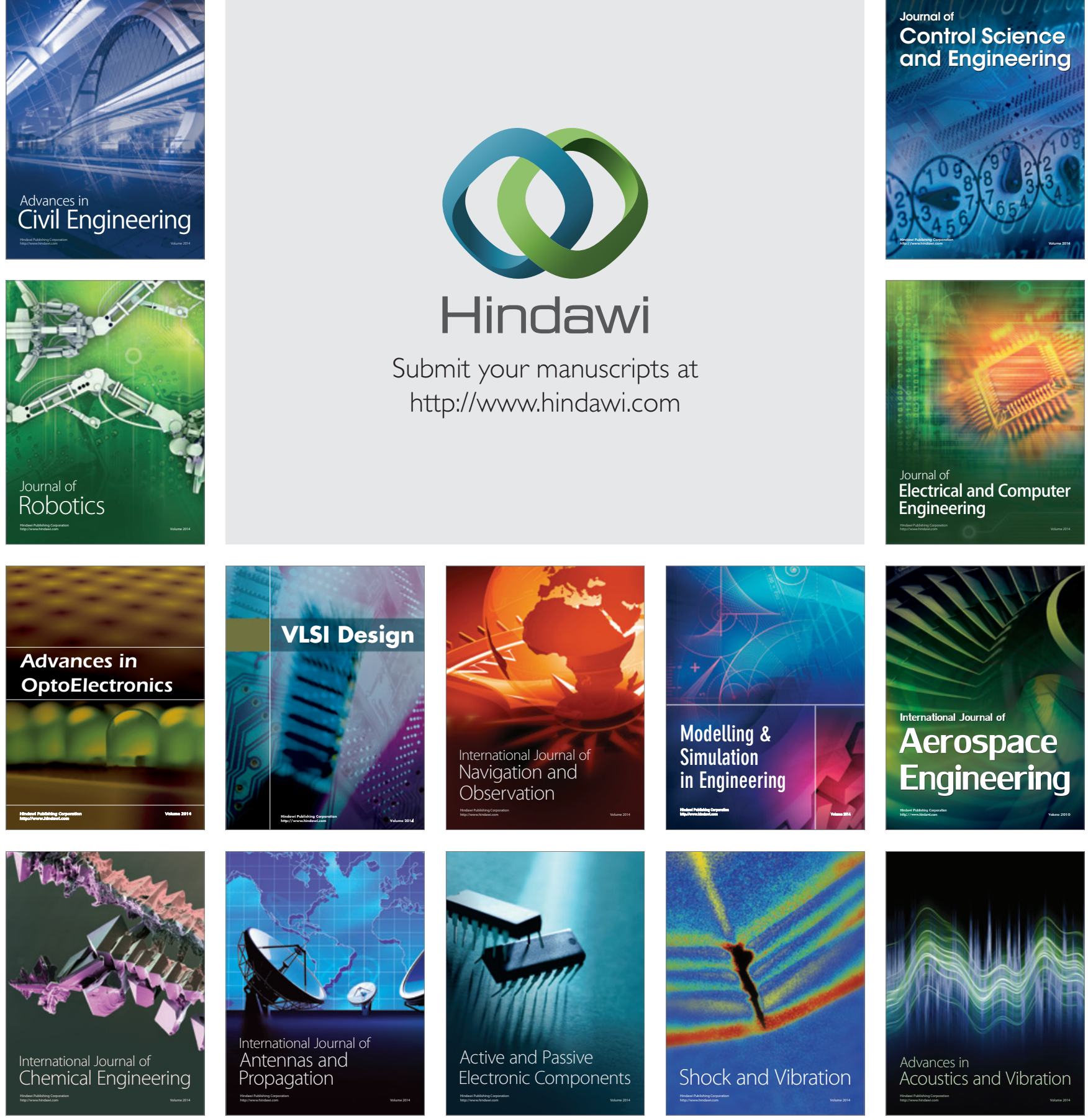